\title{
La Swiss Clinical Trial Organisation publie des directives de qualité
}

\section{Maya Grüniga , \\ Claudia Weiss ${ }^{\mathrm{b}}$, \\ Peter Meier-Abt ${ }^{\mathrm{c}}$}

a Manager Assurance qualité, Swiss Clinical Trial Organisation

b Dr, Directrice, Swiss Clinical Trial Organisation

c Prof. Dr, Président, Swiss Clinical Trial Organisation
Correspondance: Swiss Clinical Trial Organisation Petersplatz 13 CH-4051 Bâle

info(at)scto.ch
La Swiss Clinical Trial Organisation (SCTO) est l'organisation faîtière de la recherche clinique en Suisse. Elle s'engage en faveur de la coordination et d'une meilleure coopération entre les centres locaux d'étude et notamment les Clinical Trial Units (CTU) des hôpitaux universitaires (Bâle, Berne, Genève, Lausanne et Zurich) et de l'Hôpital cantonal de Saint-Gall; ces points portent essentiellement sur la spécification des standards de qualité, la formation prégraduée et postgraduée ainsi que la médiation nationale et internationale.

La SCTO a élaboré un concept transdisciplinaire de garantie de la qualité, en collaboration avec l'ensemble des CTU et le Groupe Suisse de Recherche Clinique sur le Cancer (SAKK): il a pour objectif d'améliorer la sécurité des patients et l'intégrité des données collectées lors d'essais cliniques. Le concept recommande à tous les Trials Units la mise en place d'un système de gestion de la qualité (QMS) axé sur les processus, comme par ex. ISO 9001:2008 ou similaire.

Un groupe de travail, dirigé par la SCTO, a élaboré les Guidelines for Good Operational Practice (GGOP) pour le réseau des CTU. Elles constituent un élément fondamental du concept de garantie de la qualité, se basent sur des directives d'autres réseaux acadé-
Les Guidelines for Good Operational Practice (GGOP) sont disponibles en anglais; elles peuvent être commandées auprès de la SCTO (info@scto.ch) sous forme de brochure imprimée ou téléchargées sur le site Internet (www.scto.ch).

tions nécessaires aux projets d'étude réalisés ou encadrés par les CTU. Le document se conforme ici au déroulement d'un projet d'étude - de la phase d'ébauche à son aboutissement, en passant par l'élaboration, la mise en ouvre et la réalisation. Chaque chapitre dresse la liste des conditions détaillées requises: critères, responsabilités, processus et objectifs.

Des contrôles périodiques du système QMS sont planifiés au sein du réseau et dans le cadre du concept de garantie de la qualité. Le lancement d'inspections de systèmes par Swissmedic a franchi un premier pas dans cette direction et permis d'obtenir, au cours de l'été 2011, une première analyse de grande valeur du système QMS présent dans tous les CTU. Ces derniers peuvent à présent mettre en place des mesures d'amélioration ciblées en s'appuyant sur les rapports d'inspection.

L'approbation des directives de bonnes pratiques a permis à la SCTO et au réseau des CTU d'atteindre

\section{L'objectif est d'améliorer la sécurité des patients et l'intégrité des données collectées lors d'essais cliniques.}

miques européens et représentent des standards de qualité, fixés conjointement pour la pratique opérationnelle dans les CTU. Les GGOP concernent non seulement le système de gestion des organisations prises individuellement, mais également la mise en œuvre de projets de recherche clinique.

La structure des directives de bonnes pratiques est double. La première partie décrit les exigences posées au système de gestion de l'organisation: structure, processus internes, ressources et activités des CTU. La deuxième partie traite du processus central lié aux prestations des CTU, à savoir la gestion des projets de recherche clinique. Ces chapitres décrivent les condi- une étape importante et de démontrer le caractère constructif de la collaboration existante au sein du réseau. Ils contribuent ainsi largement à l'harmonisation et à l'amélioration continue de la qualité des prestations dans le réseau des CTU, entraînant la reconnaissance de la compétence technique des CTU sur une base nationale et internationale. L'application de ces directives par d'autres centres d'étude et réseaux cliniques (existants ou en création) correspondrait à une étape importante en direction d'une amélioration et d'une harmonisation de la culture académique en Suisse. 